University of Nebraska - Lincoln

DigitalCommons@University of Nebraska - Lincoln

Faculty Publications from the Center for Plant

Science Innovation

Plant Science Innovation, Center for

November 2000

\title{
Transgene and Transposon Silencing in Chlamydomonas reinhardtii by a DEAH-Box RNA Helicase
}

\author{
Dancia Wu-Scharf \\ University of Nebraska - Lincoln \\ Byeong-Ryool Jeong \\ University of Nebraska - Lincoln, bjeong2@unl.edu \\ Chaomei Zhang \\ University of Nebraska - Lincoln \\ Heriberto D. Cerutti \\ University of Nebraska - Lincoln, hcerutti1@unl.edu
}

Follow this and additional works at: https://digitalcommons.unl.edu/plantscifacpub

Part of the Plant Sciences Commons

Wu-Scharf, Dancia; Jeong, Byeong-Ryool; Zhang, Chaomei; and Cerutti, Heriberto D., "Transgene and Transposon Silencing in Chlamydomonas reinhardtii by a DEAH-Box RNA Helicase" (2000). Faculty Publications from the Center for Plant Science Innovation. 3.

https://digitalcommons.unl.edu/plantscifacpub/3

This Article is brought to you for free and open access by the Plant Science Innovation, Center for at DigitalCommons@University of Nebraska - Lincoln. It has been accepted for inclusion in Faculty Publications from the Center for Plant Science Innovation by an authorized administrator of DigitalCommons@University of Nebraska Lincoln. 
R E P O R T S

\title{
Transgene and Transposon Silencing in Chlamydomonas reinhardtii by a DEAH-Box RNA Helicase
}

\author{
Dancia Wu-Scharf, Byeong-ryool Jeong, Chaomei Zhang, \\ Heriberto Cerutti*
}

\begin{abstract}
The molecular mechanism(s) responsible for posttranscriptional gene silencing and RNA interference remain poorly understood. We have cloned a gene (Mut6) from the unicellular green alga Chlamydomonas reinhardtii that is required for the silencing of a transgene and two transposon families. Mut6 encodes a protein that is highly homologous to RNA helicases of the DEAH-box family. This protein is necessary for the degradation of certain aberrant RNAs, such as improperly processed transcripts, which are often produced by transposons and some transgenes.
\end{abstract}

In plants (1-5) and some fungi $(6,7)$, the overexpression or misexpression of transgenes can induce the silencing of homologous sequences (i.e., transgene and endogenous gene sequences) by a process known as posttranscriptional gene silencing (PTGS). The introduction of double-stranded RNA (dsRNA) triggers a similar phenomenon, called RNA interference (RNAi), in a variety of invertebrate and vertebrate species $(7-15)$. The widespread occurrence of these phenomena in eukaryotes suggests the involvement of one or more ances-

School of Biological Sciences and Plant Science Initiative, University of Nebraska-Lincoln, E211 Beadle Center, Post Office Box 880666, Lincoln, NE 68588 , USA.

*To whom correspondence should be addressed. Email: hcerutti1@unl.edu tral mechanisms that may have evolved to limit the expression of parasitic elements, such as transposons and viruses $(3-8,10)$. Although several models have been proposed to explain PTGS and RNAi $(1,3,5,7,8,10,13,15)$, and some genes that are essential for these processes have been identified $(4-6,8-11)$, the molecular machinery responsible for RNA recognition and degradation remains largely unexplored.

To gain insight into the molecular mechanism(s) of PTGS, we have screened for mutants defective in this process in Chlamydomonas reinhardtii. In Chlamydomonas, the $R b c S 2:: a a d A: \because R b c S 2$ transgene is silenced at both transcriptional and posttranscriptional levels $(16,17)$. This transgene is composed of the coding sequence of the eubacterial aadA gene (conferring spectinomycin resis- 
tance) fused to the regulatory regions of the Chlamydomonas RbcS2 gene (encoding the small subunit of ribulose-1,5-bisphosphate carboxylase-oxygenase) (17). Strain 33$\mathrm{P}[300]$ contains a posttranscriptionally silenced copy of $R b c S 2: \because a a d A: \because R b c S 2$. By ran-
A

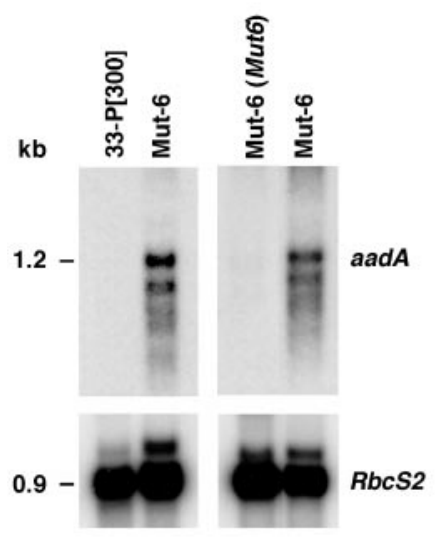

B

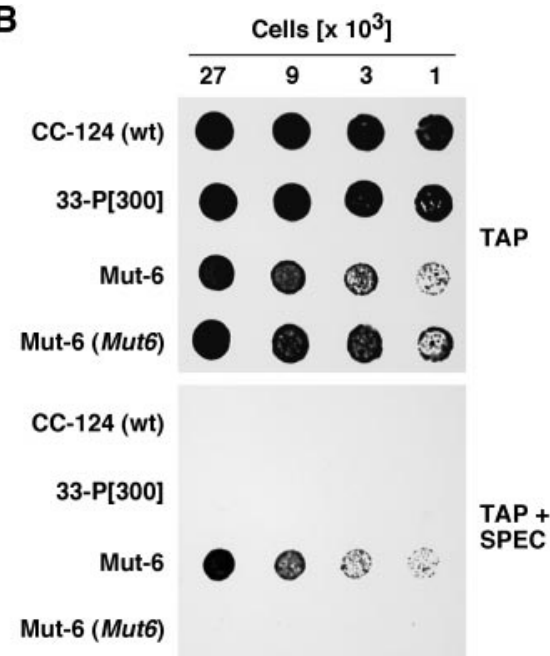

Fig. 1. Expression of the RbcS2::aadA::RbcS2 transgene is reactivated in Mut-6. (A) Northern blot analysis of the silenced strain (33-P[300]), the mutant strain (Mut-6), and the mutant strain complemented with a wild-type copy of Mut6 [Mut-6(Mut6)]. Polyadenylated RNA [poly(A) ${ }^{+}$RNA] from the indicated strains was sequentially probed with the coding sequence of aadA (upper panels) and with the coding sequence of RbcS2 (lower panels). (B) Growth and survival on tris-acetatephosphate (TAP) medium or on TAP medium containing spectinomycin (TAP + SPEC) of the indicated strains. CC-124 is the wild-type strain. Cells grown mixotrophically were diluted to the indicated number of cells per $5 \mu \mathrm{l}$, spotted on the plates, and incubated for 15 days (16).

A

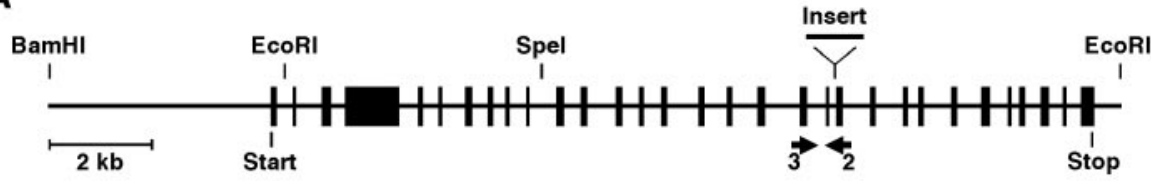

B

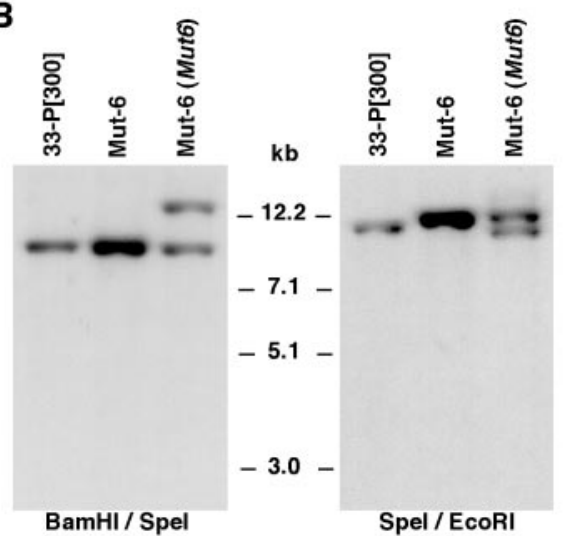

C

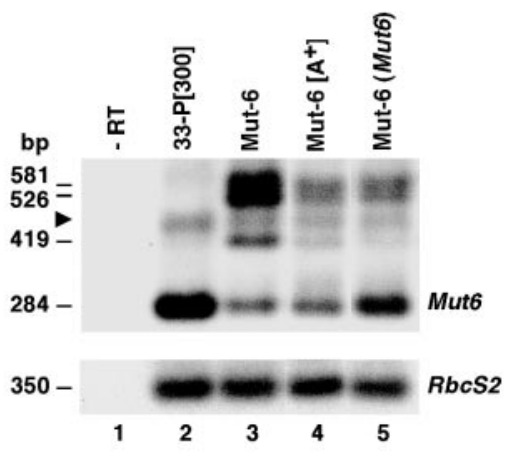

Fig. 2. Structure and expression of Mut6. (A) Genomic organization of the Mut6 gene. Exons are indicated as solid boxes. The site of insertion of the tagging plasmid in Mut- 6 is shown above the diagram. Specific primers 2 and 3 anneal to exons 21 and 19. (B) Southern blot analysis of the indicated strains. Total cell DNA was digested with either Bam HI-Spe I (left panel) or Spe I-Eco $\mathrm{RI}$ (right panel) and probed with PCR fragments corresponding to exon 8 or exons 19 through 21 respectively. The Mut- 6 lanes were loaded with twice the amount of DNA of the other lanes. (C) Analysis of Mut6 expression by RT-PCR. The upper panel shows a Southern blot of RT-PCR products amplified with Mut6 primers 2 and 3 (18). Amplification of the RbcS2 mRNA was used as a control for equal amounts of input RNA and for the efficiency of the RT-PCRs (lower panel). Total RNA (lanes 1, 2, 3, and 5) or poly (A) ${ }^{+}$RNA (lane 4) was used as template for RT-PCR (18). The input of poly $(A)^{+}$RNA was adjusted to obtain an amplification of $R b c S 2$ equivalent to that in the total RNA samples. A nonspecific RT-PCR fragment is seen across lanes 2 through 5 (upper panel, arrowhead). - RT, no reverse transcriptase.

dom insertional mutagenesis (18), we isolated a mutant strain (Mut-6) that grew on spectinomycin-containing medium (19). Standard genetic analyses confirmed that the gene disrupted in Mut- 6 is required for silencing of the transgene (18).

We next examined the expression of the $R b c S 2: \because a a d A: \because R b c S 2$ transgene by Northern blot analysis. Hybridization to the $a a d A$ coding sequence was observed in samples from Mut- 6 but was undetectable in those from 33-P[300] (Fig. 1A). The expected size of the $R b c S 2: \because a a d A: \because R b c S 2$ mRNA is $\sim 1.2 \mathrm{~kb}$, and the smaller transcripts are thought to correspond to improperly processed RNAs that have undergone removal of a cryptic intron, premature termination, and/or incorrect polyadenylation (17). Consistent with the steady-state levels of transgenic transcripts, Mut-6 grew on selective medium whereas 33-P[300] could not survive (Fig. 1B). The mutant strain also showed a slower growth rate than the parental strain (Fig. 1B, upper panel). In addition, Mut-6 was moderately sensitive to treatment with two genotoxic agents, methyl methanesulfonate (MMS) and bleomycin, but showed wild-type sensitivity to ultraviolet irradiation (19). These pleiotropic phenotypes suggest that Mut6 plays a role in other cellular processes in addition to transgene silencing.

To clone the Mut6 gene, we obtained genomic sequences flanking the tagging plasmid (Fig. 2A) by asymmetric polymerase chain reaction (PCR) with nested plasmid primers and an arbitrary degenerate primer. The PCR fragments were used as probes to screen a genomic cosmid library, and one of the isolated cosmids was able to complement the defective phenotypes of Mut-6 (18). The complemented strain Mut-6(Mut6) was unable to grow on medium containing spectinomycin (Fig. 1B), and the $R b c S 2: \because a a d A \because: R b c S 2$ transcript was almost undetectable by RNA hybridization (Fig. 1A). In Southern blots of total cell DNA, all strains had a 9.5-kb Bam HI-Spe I fragment, encompassing the $5^{\prime}$ end of Mut6. Mut6(Mut6) also showed a larger fragment (Fig. 2B) that resulted from deletion, presumably during integration into the nuclear genome, of cosmid DNA upstream of Mut6 and digestion within sequences flanking the insertion site (19). An 11-kb Spe I-Eco RI fragment, corresponding to the $3^{\prime}$ end of Mut6, was restored in the complemented strain. This segment is approximately $1.1 \mathrm{~kb}$ larger in Mut- 6 because of insertion of the tagging plasmid near the $3^{\prime}$ end of intron 20 (Fig. 2B) (18). Thus, Mut-6(Mut6) contains one mutant copy of the endogenous Mut6 gene and one complementing wild-type copy.

We analyzed the expression of Mut6 by reverse transcriptase PCR (RT-PCR) with primers annealing to exons 19 and 21 (18), flanking the site of insertion of the tagging plasmid (Fig. 2A). When total RNA was used 


\section{R E P O R T S}

as template, Mut- 6 showed a greater than fivefold reduction in the amount of the expected PCR product [a 284-base pair (bp) fragment] when compared with the parental strain (Fig. 2C). It also showed three additional PCR products of 419, 526, and 581 bp (Fig. 2C), containing tagging vector sequences improperly spliced as exons (18). These misspliced transcripts were mostly nonpolyadenylated (Fig. $2 \mathrm{C}$; compare Mut- 6 with Mut- $6\left[\mathrm{~A}^{+}\right]$) and were presumably not translated. Thus, the mutant phenotypes are likely caused by a reduction in the amount of wild-type protein (18). The complemented strain partially restored the level of the wild-type PCR product and showed a decrease in the amount of improperly processed transcripts [Fig. 2C; compare Mut-6 and Mut6(Mut6)]. Because Mut-6(Mut6) integrated a single copy of Mut 6 without altering the mutant locus (Fig. 2B), these results suggested that the Mut6 protein might be involved in the degradation of the misprocessed aberrant RNAs.

As observed for several RNAi-resistant mutants in Caenorhabtidis elegans $(8-10,13)$, Mut-6 shows elevated transposition activity. We examined the expression of a retrotransposonlike element, TOC1 (20), in the mutant and wild-type strains. The steady-state level of TOC1 RNA, which is mostly nonpolyadenylated and heterogeneous in size (20), is about threefold higher in Mut-6 as compared with the parental and complemented strains (Fig. 3A). The transposition frequency of $T O C 1$ is accordingly enhanced in Mut- 6 . We detected no obvious differences in the copies of TOC1 integrated into the genome of 33-P[300] after 1 year of growth (Fig. 3B; compare 33-P[300] and 33-P[300]). In contrast, all parallel cultures of the mutant strain showed additional TOC1 copies (Fig. 3B, arrowheads). The transposition activity of another element that moves via a DNA intermediate, Gulliver (21), was also higher in Mut-6 (Fig. 3C).

The Mut6 gene was sequenced entirely from the complementing cosmid clone. The coding sequence was confirmed by RT-PCR with primers designed to anneal to predicted exons (19). The deduced Mut6 protein contains 1431 amino acids and is a member of the DEAH-box RNA helicase family (Fig. 4). Within this family, Mut6 $p$ is most similar to human PRP16, a homolog of an essential pre-mRNA splicing factor in Saccharomyces cerevisiae, and to MOG-1, a gene involved in sex determination in C. elegans (22) (Fig. 4A). Mut6p possesses, in a region spanning residues 751 to 1066 , all amino acid motifs that are shared by the DEAH-box family of RNA helicases (23) (Fig. 4B). In addition, Mut6p has a glycine-rich region spanning residues 150 to 460 . This domain includes several RGG repeats (24) resembling an RGG box, a motif implicated in RNA binding and protein-protein interactions (25). Mut6p also has three putative nuclear localization signals and is predicted to be nuclear by both
PSORT (a program for detecting sorting signals and predicting subcellular protein localization) and neural network analyses (26).

Because Mut $6 p$ is similar to general splicing factors, we tested whether the mutant strain showed a defect in pre-mRNA splicing. We examined the transcripts of TubA (encoding $\alpha 1$ tubulin) and $R b c S 2$ by both Northern blot hybridization of total RNA and RT-PCR with specific primers. In neither case could we detect unspliced products (18), which suggests that Mut6 is not essential for general splicing. However, Mut-6 showed larger amounts of RNAs

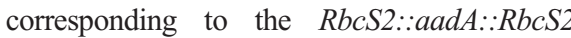
transgene (Fig. 1A) and to the retroelement TOC1 (Fig. 3A). It also showed improperly processed Mut6 transcripts as a result of the tagging plasmid insertion (Fig. 2C). All of these RNAs appeared to be aberrant, with defects in proper processing such as splicing and/or polyadenylation. In the complemented strain, the levels of these RNAs were markedly reduced, suggesting that the Mut6 RNA helicase might be involved in the degradation of abnormal RNAs. To directly test the role of Mut6 in the turnover of TOC1 RNA, transcription was inhibited with actinomycin D and the stability of the retrotransposon transcripts was examined during a 3-hour time course (18). In the parental strain, we clearly observed decay of TOC1 RNA followed by stabilization at the later time points (Fig. 3, D and E). This time course is similar to that reported for short interspersed nuclear elements (SINE) transcribed by RNA

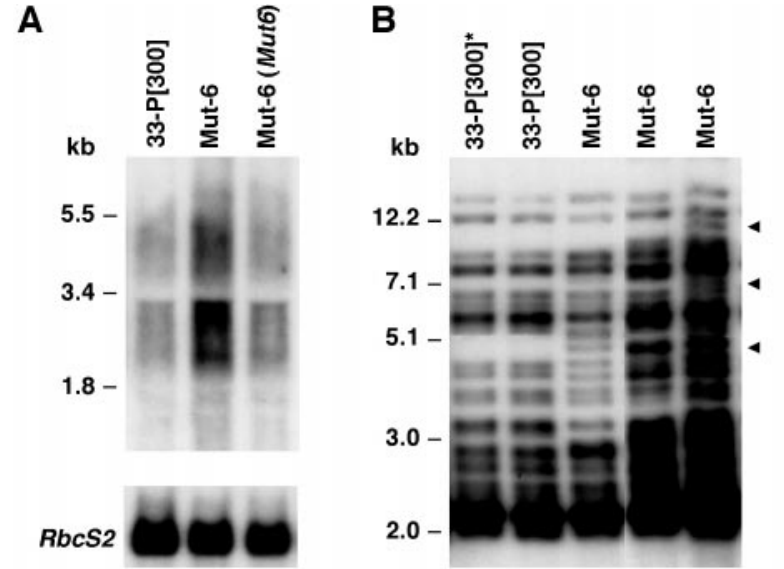

D

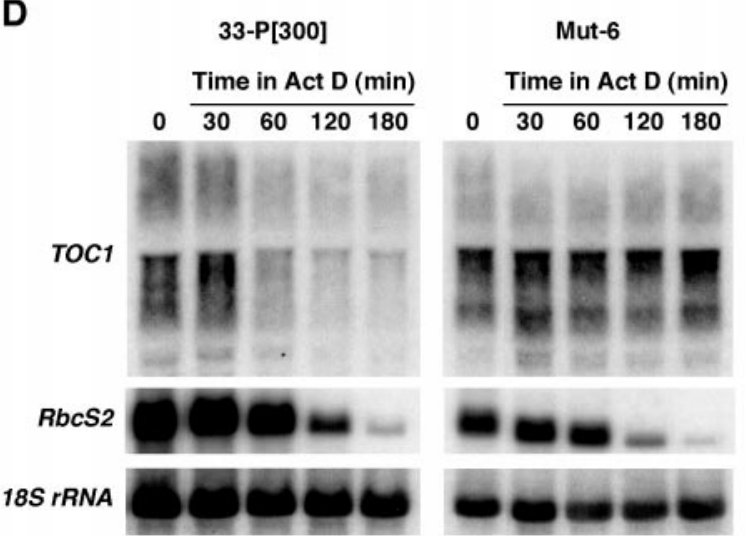

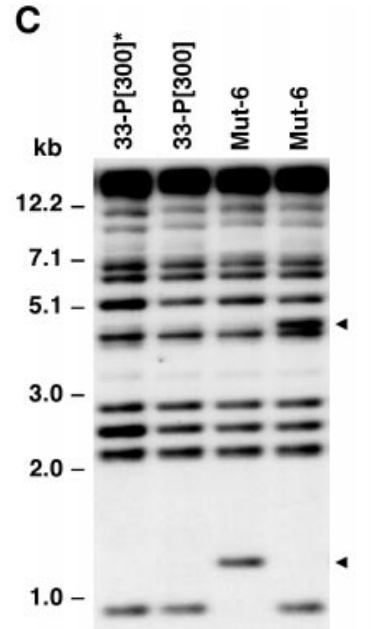

E

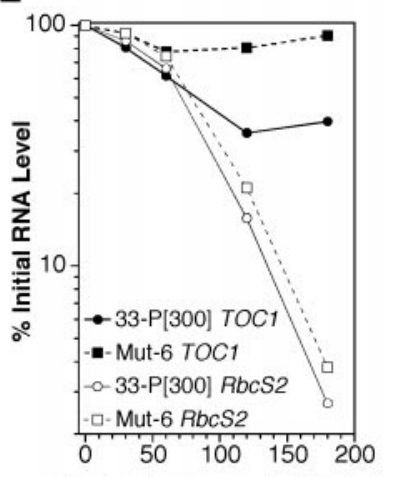

Time in Actinomycin D ( $\mathrm{min})$

Fig. 3. Mut6 effects on the activity of a retroelement, TOC1, and a DNA transposon, Gulliver. (A) Northern blot of total RNA probed sequentially for TOC1 (upper panel) and for RbcS2 (lower panel). (B) Southern blot analysis of TOC1 transposition. DNA from the silenced strain was isolated and stored frozen (33-P[300]*). Parallel cultures of 33-P[300] and Mut-6 were then grown for 1 year and DNA was isolated again. Total cell DNA was digested with Hinc II and probed for TOC1. The arrowheads indicate several new fragments in the subclones of Mut-6. (C) Southern blot analysis of Gulliver transposition. Parallel cultures of 33-P[300] and Mut- 6 were grown as described above. Total cell DNA was digested with Hind III and probed for Gulliver. The arrowheads indicate fragments with altered mobility in the subclones of Mut-6. (D) Northern blot analysis of RNA decay in 33-P[300] and Mut-6. Total RNA, isolated at the indicated times after the addition of actinomycin D (Act D), was probed sequentially for TOC1 (upper panel), for RbcS2 (middle panel), and for 185 rRNA (lower panel). Ten micrograms of total RNA were loaded per lane for 33-P[300] and $4 \mu \mathrm{g}$ of total RNA were loaded per lane for Mut-6. (E) Semilog plot of the kinetics of RNA decay after addition of actinomycin D. RNA amounts were determined by phosphorimager analysis of Northern blots, and differences in loading were corrected by normalizing to the levels of $18 \mathrm{~S}$ rRNA. Each time point represents the average of two independent experiments. 
polymerase III (27). The mutant strain Mut-6 showed a much reduced decay of TOC1 transcripts, followed by earlier stabilization (Fig. 3, $\mathrm{D}$ and $\mathrm{E})$. The initial rate differences in TOC1 RNA turnover (2.5-fold) are sufficient to account for the different levels of TOCl transcripts in 33-P[300] and Mut-6. In contrast, the decay of a correctly processed message, such as $R b c S 2$, was virtually identical in the parental and mutant strains (Fig. 3, D and E).

Although Mut6p does not appear to function in general splicing (18), it could still be involved in the processing of a subset of specific mRNAs whose products are required in various cellular processes. However, the simplest interpretation of our results is that Mut6p participates directly in RNA degradation. It could have at least two, not mutually exclusive, roles. (i) Mut6p could be a component of the machinery responsible for PTGS and the turnover of homologous RNAs. This process could be induced by dsRNA because antisense transcripts to TOC1 (20) and to RbcS2::aadA::RbcS2 (19) have been detected. (ii) Mut6p could be part of an RNA surveillance system that recognizes and degrades improperly processed RNAs. This might explain the sensitivity of Mut- 6 to MMS and bleomycin, as Mut6p may be necessary to degrade damaged and faulty messages (28).

It appears that eukaryotes have evolved a network of mRNA surveillance systems that degrades aberrant RNAs (29). These systems might also operate to control the expression of rogue genes encoded by viruses, transposable elements, and some transgenes, because they often produce abnormal RNAs, including anti-

\section{A}
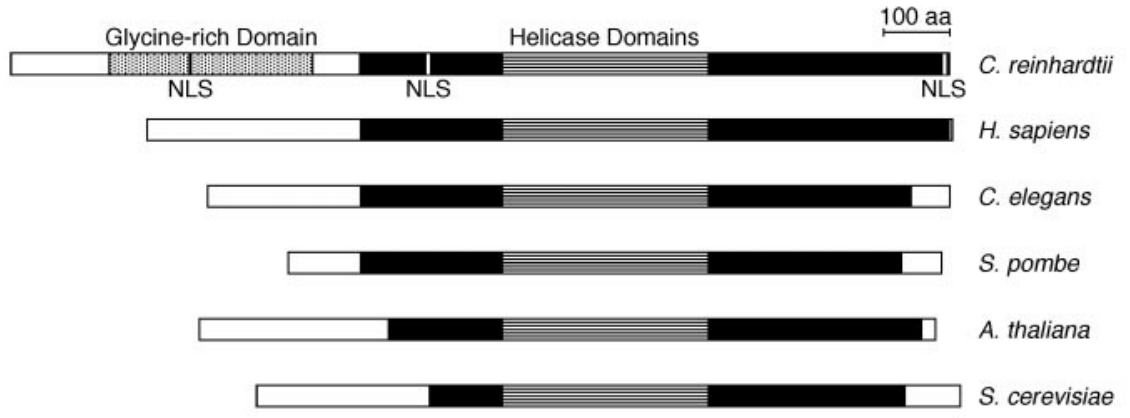

B
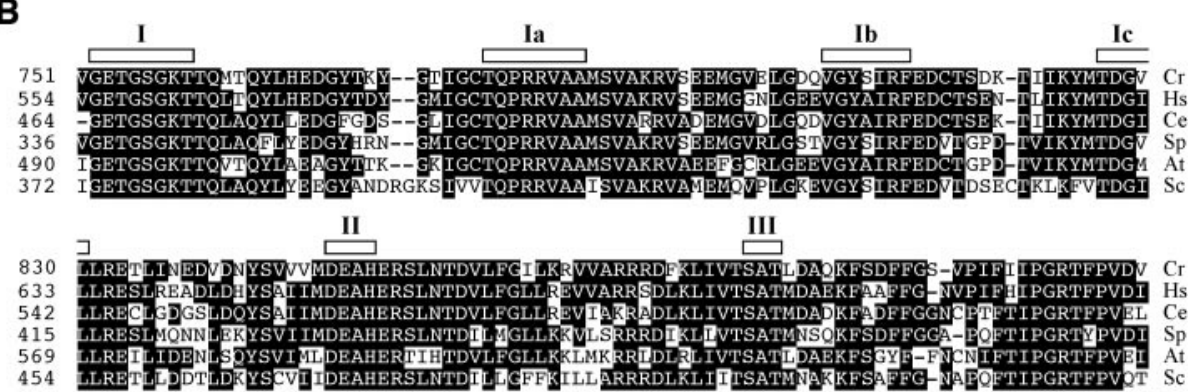

454 LLRETLLDDTLDKYSCVIIDEAHERSLNTDILIGFFKILLARRRDLKLITISATMNARKFSAFFG- NAFOFTIPGRTFPVOT SC
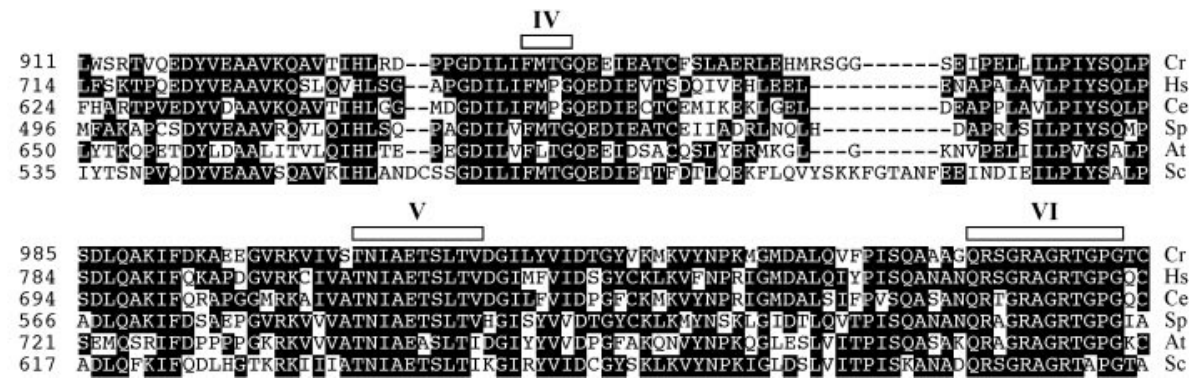

Fig. 4. Mut6 belongs to the DEAH-box RNA helicase family. (A) Schematic representation of Mut6p and five closely related DEAH-box RNA helicases. The sequences correspond to hPRP16 (Homo sapiens, AAC27431), MOG-1 (C. elegans, P34498), a putative pre-mRNA splicing factor (Schizosaccharomyces pombe, CAB52799), DDX8 (Arabidopsis thaliana, Q38953), and PRP16 (S. cerevisiae, NP_013012). The solid areas indicate aligned regions with greater than $45 \%$ identity. The helicase domains are shown as horizontally striped boxes. Putative nuclear localization signals (NLS) and a unique glycine-rich domain (shaded box) are indicated in the diagram of Mut6p. The coding sequence of Mut6 has been deposited in GenBank (accession no. AF305070). (B) Sequence alignment of the helicase domains of Mut6p $(\mathrm{Cr})$ and the five related DEAH-box proteins (Hs, hPRP16; Ce, MOG-1; Sp, S. pombe putative splicing factor; At, DDX8; and Sc, PRP16) (24). Boxes above the sequences indicate the positions of conserved helicase domains (23). sense RNA and dsRNA $(1,3,5,7,8,10)$. Although dsRNA is the initiator of RNAi, a signal other than dsRNA may be used to detect and suppress transposon activity in C. elegans (13). RNAs that are not necessarily doublestranded have also been implicated in PTGS in plants $(1,3,5,30)$. One possibility is that multisubunit complexes, perhaps analogous to the $S$. cerevisiae exosome $(10,31)$, degrade a variety of aberrant RNAs and dsRNA-targeted transcripts through the action of different adaptors recognizing specific features of the target RNAs. It is tempting to speculate that the Chlamydomonas Mut6p might be involved in such a process.

\section{References and Notes}

1. R. A. Jorgensen, Q. D. Que, M. Stam, Trends Genet. 15, 11 (1999).

2. A. J. Hamilton, D. C. Baulcombe, Science 286, 950 (1999).

3. J. M. Kooter, M. A. Matzke, P. Meyer, Trends Plant Sci. 4, 340 (1999).

4. P. Mourrain et al., Cell 101, 533 (2000).

5. T. Dalmay, A. Hamilton, S. Rudd, S. Angell, D. Baulcombe, Cell 101, 543 (2000).

6. C. Cogoni, G. Macino, Science 286, 2342 (1999).

7. A. Fire, Trends Genet. 15, 358 (1999).

8. R. F. Ketting, T. H. A. Haverkamp, H. G. A. M. van Luenen, R. H. A. Plasterk, Cell 99, 133 (1999).

9. H. Tabara et al., Cell 99, 123 (1999).

10. J. M. Bosher, M. Labouesse, Nature Cell Biol. 2, E31 (2000).

11. A. Smardon et al., Curr. Biol. 10, 169 (2000).

12. R. F. Ketting, R. H. A. Plasterk, Nature 404, 296 (2000).

13. A. Grishok, H. Tabara, C. C. Mello, Science 287, 2494 (2000).

14. S. M. Hammond, E. Bernstein, D. Beach, G. J. Hannon, Nature 404, 293 (2000).

15. P. D. Zamore, T. Tuschl, P. A. Sharp, D. P. Bartels, Cell 101, 25 (2000).

16. H. Cerutti, A. M. Johnson, N. W. Gillham, J. E. Boynton, Plant Cell 9, 925 (1997).

17. Genetics 145, 97 (1997)

18. For experimental details and supplemental material, see Science Online at www.sciencemag.org/feature/ data/1051322.shl.

19. D. Wu-Scharf, H. Cerutti, unpublished data.

20. A. Day, J. D. Rochaix, J. Mol. Biol. 218, 273 (1991).

21. P. J. Ferris, Genetics 122, 363 (1989).

22. A. Puoti, J. Kimble, Mol. Cell. Biol. 19, 2189 (1999).

23. E. Jankowsky, A. Jankowsky, Nucleic Acids Res. 28, 333 (2000).

24. Single-letter abbreviations for the amino acid residues are as follows: A, Ala; C, Cys; D, Asp; E, Glu; F, Phe; G, Gly; H, His; I, Ile; K, Lys; L, Leu; M, Met; N, Asn; P, Pro; Q, Gln; R, Arg; S, Ser; T, Thr; V, Val; W, Trp; and $Y$, Tyr.

25. A. M. Krecic, M. S. Swanson, Curr. Opin. Cell Biol. 11, 363 (1999).

26. K. Nakai, P. Horton, Trends Biochem. Sci. 24, 34 (1999).

27. R. H. Kimura, P. V. Choudary, C. W. Schmidt, Nucleic Acids Res. 27, 3380 (1999).

28. S. M. Hecht, J. Nat. Prod. 63, 158 (2000).

29. P. Hilleren, R. Parker, Annu. Rev. Genet. 33, 229 (1999).

30. M. Metzlaff, M. O'Dell, R. Hellens, R. B. Flavell, Plant J. 23, 63 (2000).

31. C. Allmang et al., Genes Dev. 13, 2148 (1999).

32. We thank J. Kovar and D. Weeks for making available plasmid pJK7 and an anonymous reviewer for helpful suggestions. Supported by grants to H.C. from NSF (grant number MCB-9808473) and from the Nebraska Research Initiative.

13 April 2000; accepted 13 September 2000 